\title{
Effect of ozone oxidative preconditioning on inflammation and oxidative stress injury in rat model of renal transplantation ${ }^{1}$
}

\author{
Zhishun Wang', Qi Han", Yong-lian Guo"I', Xiu-heng Liu'v , Tao Qiuv
}

'PhD, Department of Urology, The Central Hospital of Wuhan, Tongji Medical College, Huazhong University of Science and Technology, Wuhan, China. Conception and design of the study, acquisition and analysis of data, manuscript writing. "Postgraduate, Department of Nephrology, The Fifth Hospital of Wuhan, Wuhan, China. Technical procedures, acquisition and interpretation of data.

IIIPhD, Physician, Department of Urology, The Central Hospital of Wuhan, Tongji Medical College, Huazhong University of Science and Technology, Wuhan, China. Design of the study, acquisition of data, supervised all phases of the study.

IVPhD, Full Professor, Department of Urology, Renmin Hospital of Wuhan University, Wuhan, China. Design of the study, critical revision.

VPhD, Physician, Department of Organ Transplantation, Renmin Hospital of Wuhan University, Wuhan, China. Acquisition of data.

\begin{abstract}
Purpose: To investigate the effect of ozone oxidative preconditioning (OzoneOP) on inflammation and oxidative stress injury in rat model of renal transplantation.

Methods: Thirty six male Sprague Dawley (SD) rats were randomly divided into three groups. Sham group: rats were treated with opening and closing abdomen. Kidney transplantation group (KT group): SD rat received the donor's left kidney derived from another SD rat. Ozone oxidative preconditioning and kidney transplantation (OOP+KT group): donor SD rats received OzoneOP treatments by transrectal insufflations before kidney transplantation. After transplantation, parameters of renal function of recipients were determined. Morphology and pathological changes of renal allograft were examined. Expression of NF-KBp65, HMGB-1 were also determined by Western-blot.

Results: Compared to KT group, the morphology and pathological damages of renal allograft were less serious in OOP+KT group. Meanwhile, levels of SOD and GSH-Px of renal allograft in OOP+KT group were higher than those in KT group respectively. Western-blot showed that the expressions of NF-KBp65 and HMGB-1 in OOP+KT group were obviously less than those in KT group.

Conclusion: Ozone oxidative preconditioning could attenuate the inflammatory reaction and oxidative stress injury in renal allograft, which might be related with the enhancement of anti-oxidative system and suppression of inflammatory reaction.
\end{abstract}

Key words: Kidney Transplantation. Ozone. Oxidative Stress. Rats. 


\section{- Introduction}

The most effective treatment measure for end-stage renal disease patients who need dialysis is still the renal transplantation. There are growing kinds of effective immunosuppressive agents which show powerful abilities on suppressing and controlling the acute rejection during the perioperative period of kidney transplantation. However, there were still many adverse factors which were difficult to avoid during the operation, and one of main disadvantage factors is ischemia reperfusion injury. Ischemia reperfusion injury involves complex pathological process, which contains many factors, such as oxidative stress injury, intracellular inflammatory response, cell apoptosis and necrosis, calcium overload and so on. Therefore, treatment measures which could attenuate the inflammation reaction and oxidative stress injury may be beneficial for the renal function recovery of renal allograft.

Ozone as one kind of powerful oxidizing gas, which is widely used to the disinfection of water and food. Some report has showed that ozone had numerous abilities, ozone therapy could be used to treat infected wound and empyrosis ${ }^{1}$. Furthermore, researches have showed that ozone therapy could enhance the function of anti-oxidative system, which could mitigate the damage coused by oxidative stress $^{2,3}$. However, to our knowledge, the effect of ozone oxidative preconditioning on inflammation and oxidative stress injury in the homologous kidney transplantation has not been studied up till now.

Therefore, the current study tried to research the effect of ozone oxidative preconditioning on inflammation and oxidative stress injury in homologous kidney transplant in rat model.

\section{Methods}

The experimental protocol was approved by the Animal Ethics Review Committee of Wuhan University, and the procedures were carried out accordance with the recommendations in the Guide for the Care and Use of Laboratory Animals of the National Institutes of Health.

Thirty six males, adult SD rats (body weight 250-300g) were acquired from the Experimental Animal Center of Medical College of Wuhan University (Wuhan, China). Animals were housed at the Central Animal Facility of Affiliated Renmin Hospital of Wuhan University based on standard guidelines. Rats were kept in an air-filtered, homoiothermal $\left(20-22^{\circ} \mathrm{C}\right)$, and light-controlled (light for 8a.m.-8p.m.) room, and allowed free access to a standard diet.

\section{Experimental protocol}

Kidney donor rats were randomly divided into three groups: OOP+KT group $(n=6)$ : before the kidney transplant procedure, donor rats received 15 OzoneOP treatments by transrectal insufflations $(1 \mathrm{mg} / \mathrm{kg})$, once a day, at an ozone concentration of $50 \mu \mathrm{g} / \mathrm{ml}$; KT group: SD rat received the donor's left kidney derived from another SD rat; Sham group: rats were treated with opening and closing abdomen. All donors were intraperitoneal with atropine $(0.01 \mathrm{mg} / \mathrm{kg})$, buprenorphine (0.04 mg/kg), diazepam (10 mg/kg). Ten minutes later, they were anesthetized with pentobarbital $(45 \mathrm{mg} / \mathrm{kg})$. Then, the donor's blood vessels and ureter were fully separated. Kidneys were flushed through the aorta with $3 \mathrm{ml}$ of $4^{\circ} \mathrm{C}$ cold Ringer lactate solution with heparin $(50 \mathrm{U} / \mathrm{ml})$ until homogeneously pale. Left kidneys were removed with vascular and ureter with an ureterocystic flap and placed in cold Ringer lactate solution at $4^{\circ} \mathrm{C}$ for 180 
min. In recipient rats, after left nephrectomy, renal arterial and venous anastomoses were performed as end-to-end anastomoses to the renal arterial and venous of recipient, respectively. Then, anastomoses of donor ureterocystic flap to recipient's bladder was constructed. Finally, recipient rats received right nephrectomy. During the surgery, body temperature was monitored and constantly kept between $35{ }^{\circ} \mathrm{C}$ and $37^{\circ} \mathrm{C}$. Animals were placed on a warm blanket with free access to water and standard laboratory chow ad libitum after transplantation. Twenty-four hours after transplantation, blood was drawn for analysis, animals were sacrificed and kidneys were harvested for different determinations.

\section{Serum assays}

To assess $\mathrm{Cr}$ and BUN, blood samples were collected, centrifuged and kept at $-20^{\circ} \mathrm{C}$ until analyses, adopting standard techniques using an Olympus AU 2700 Analyzer (Olympus Optical Co. Ltd., Tokyo, Japan).

\section{Histological examination}

The kidney was fixed in $10 \%$ neutralbuffered formalin, paraffin embedded and sectioned at 4- $\mu \mathrm{m}$ thick according to standard procedure. Sections were deparaffinized and hydrated gradually and examined by $H \& E$ staining. Morphological assessment was performed by an experienced renal pathologist who was unaware of the treatment. A grading scale of $0-4$, as outlined by Jablonski ${ }^{4}$, was used for the histopathological assessment of isogeneic renal transplantation induced damage of the renal proximal tubules.

\section{Periodic Acid Schiff (PAS) Staining}

Serial sections (thickness, $4 \mu \mathrm{m}$ ) were washed with distilled water, incubated in
0.5-1 \% (v/v) aqueous periodate for 5-10 min, and washed three times with distilled water. Sections were differentiated, followed by incubation in Schiff 's reagent for 10-30 min. After staining, sections were subjected to washing three times with sulfite, then with distilled water. Sections were counterstained with hematoxylin to identify nuclei.

\section{Immunohistochemistry}

The expression of NF-kBp65, HMGB1 were conducted by immunohistochemical staining. Briefly, $5-\mu \mathrm{m}$ sections were deparaffinized, and endogenous peroxidase activity was blocked with $3 \%$ hydrogen peroxide at $37^{\circ} \mathrm{C}$ for $10 \mathrm{~min}$. Then, the sections were treated with $10 \%$ normal goat serum (Boster Biological Technology, Ltd., Wuhan, China) in Tris-buffered saline (TBS) for $30 \mathrm{~min}$ at $37^{\circ} \mathrm{C}$. Subsequently, they were incubated overnight at $4^{\circ} \mathrm{C}$ with the rabbit polyclonal anti-rat antibody (NF-KBp65; dilution at 1:300; Santa Cruz Biotechnology, Santa Cruz, CA) and rabbit polyclonal anti-rat antibody (HMGB1; dilution at 1:1000; Proteintech Group, Wuhan, China). After washing three times with phosphate-buffered saline (PBS), these sections were incubated with the HRPconjugated anti-rabbit secondary antibody (dilution at 1:5000, Boster, Wuhan, China) for $30 \mathrm{~min}$ at room temperature, followed by color reagent 3,3'-diaminobenzidine (DAB). For the negative control group, the procedures were performed with the exception of the addition of the primary antibody.

\section{Enzyme-Linked Immunosorbent Assay}

Detecting the levels of serum IL-6, IL-18 and COX-2 strictly according to the instructions of ELISA kits produced by Elabscience Biotechnology Co.,Ltd. 
Measurement of MDA, SOD and GSH-Px in Kidney

Renal tissue malondialdehyde (MDA) concentration was measured by the thiobarbituric acid (TBA) method. Amounts of lipid peroxides (LPO) were measured as the production of MDA. Absorbance was measured at $532 \mathrm{~nm}$ using a spectrometer (assay kit; Nanjing Jiancheng Bioengineering Institute). Superoxide dismutase (SOD) activity in renal tissue was measured using a commercialized chemical assay kit (Nanjing Jiancheng Bioengineering Institute) by the xanthine oxidase method.

Absorbance was determined at $550 \mathrm{~nm}$ using a spectrometer. Glutathione peroxidase (GSH-Px) activity was determined by the colorimetric method using a GSH-Px kit (Nanjing Jiancheng Bioengineering Institute). Absorbance was determined at $412 \mathrm{~nm}$ using a spectrometer. All protein concentrations of renal tissue homogenate samples were determined with Coomassie blue method (assay kit; Nanjing Jiancheng Bioengineering Institute).

\section{Western Blot analysis}

Proteins were extracted and purificated from renal tissue as previously described. In brief, protein samples were prepared for gel electrophoresis to be separated on $12.5 \%$ sodium dodecyl sulfate-polyacrylamide gels $(40 \mu \mathrm{g} / \mathrm{lane})$ and then transferred to a nitrocellulose membrane (Bio-Rad). The membrane was blocked with $5 \%$ nonfat dry milk in TBST buffer and then incubated with primary antibodies overnight at homoiothermy of $4^{\circ} \mathrm{C}$. After rinsing with TBST buffer extensively, the blots were incubated with secondary antibodies, and developed with the use of an enhanced chemiluminescence system (ECL kit; Pierce Biotechnology Inc., Rockford, IL) and captured on light-sensitive imaging film (Kodak) to analyze. The following kits were used as primary antibodies: the rabbit polyclonal antiNF-kBp65 (1:300; Santa Cruz, CA), HMGB1 (1:1000; Proteintech Group, Wuhan, China). HRP-conjugated anti-rabbit or anti-mouse secondary antibodies (Boster, Wuhan, China) was used as secondary antibody.

\section{Statistical analysis}

All data was statistically analyzed using the statistical package for the social sciences (SPSS) version 18.0 (SPSS Inc., Chicago, IL, USA). The means of the different groups were compared using the Student's t-test and differences were considered statistically significant when $p<0.05$.

\section{Results}

Effect of ozone oxidative preconditioning on renal function after renal transplantation

The renal function parameters of rats were detected at $24 \mathrm{~h}$ after renal transplantation. Rats subjected to isogeneic renal transplantation showed significant increase in blood urea nitrogen and serum creatinine compared with sham operated rats. Though the renal function changes induced by renal transplantation were slightly improved by ozone oxidative preconditioning, there were no significant differences between group KT and OOP+KT on the levels of BUN and serum $\mathrm{Cr}$ (Figure 1). 


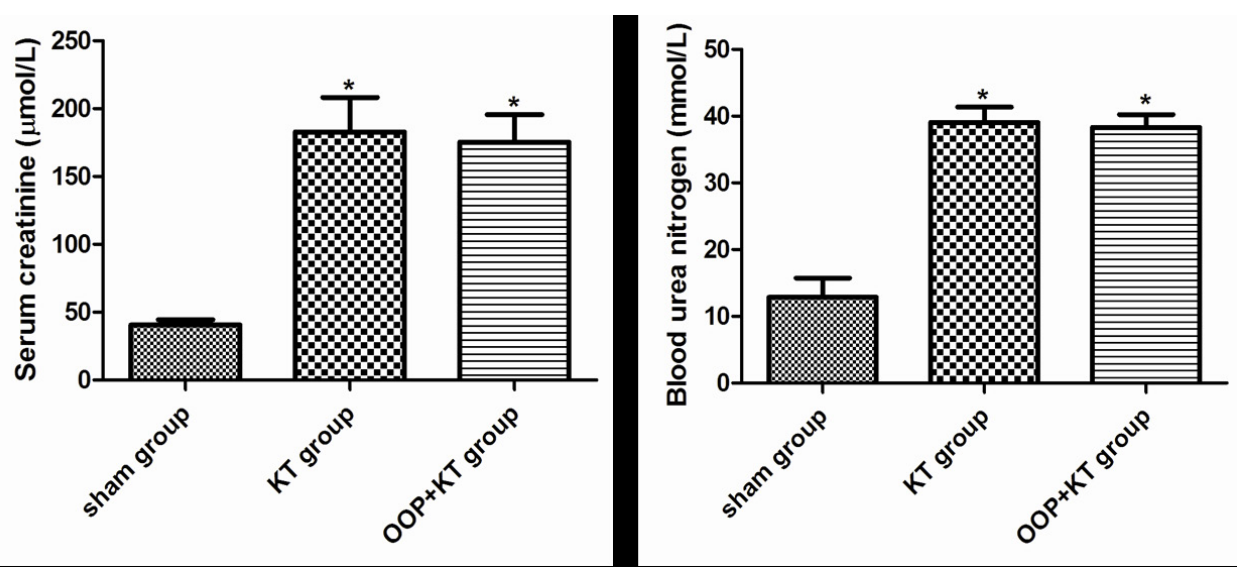

Figure 1 - Effects of ozone oxidative preconditioning on the renal function after kidney transplantation in rat model. Both serum $\mathrm{Cr}$ and BUN concentrations in groups KT and OOP+KT were significantly higher than those in group sham. Though the levels of serum $\mathrm{Cr}$ and $\mathrm{BUN}$ in OOP+KT group were slightly lower than those in group KT, there were no significant differences in the levels of serum $\mathrm{Cr}$ and BUN between groups KT and OOP+KT. ${ }^{*} p<0.05$ vs. sham group.

\section{Effect of ozone oxidative preconditioning} on morphological lesions after renal transplantation

\section{Histopathological}

examination revealed that morphological lesions existed in the allograft kidney tissue after isogeneic renal transplantation. However, OzoneOP could alleviate the extent of renal morphology damages. The isogeneic renal transplantation resulted in significant renal injury as evidenced by loss of brush border, tubular cell swelling and necrosis, tubular dilation. However, these renal damages could be attenuated by OzoneOP (Figures 2 and 3). Furthermore, there were less renal tubular necrotizing changes in the OOP+KT group than those in KT group. The Jablonski grade analysis of severe acute tubular necrosis also showed that Jablonski grade in $\mathrm{OOP}+\mathrm{KT}$ group was obviously lower than that in $\mathrm{KT}$ group (Figure 4).

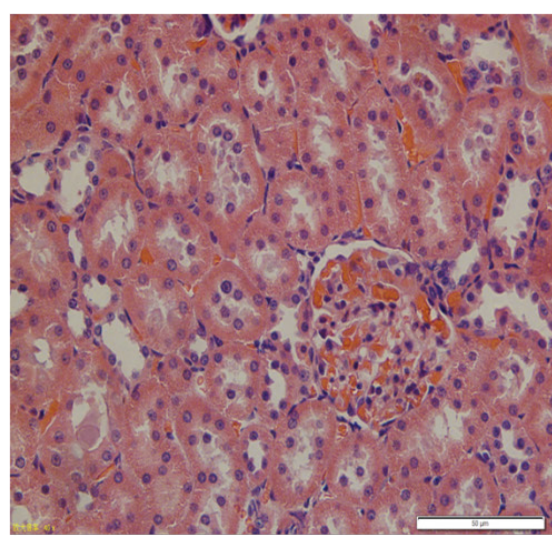

Sham group

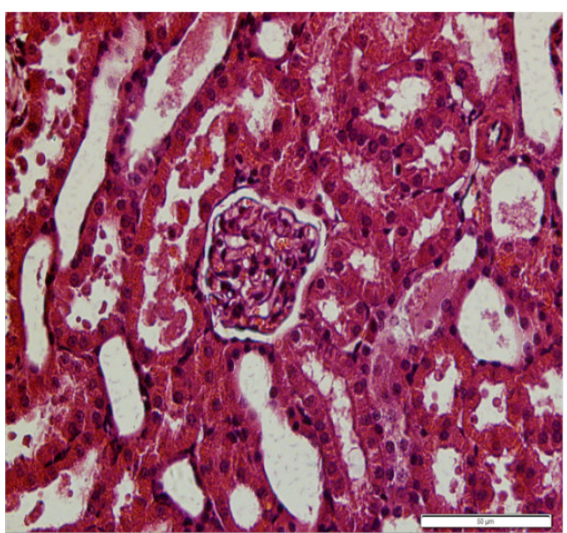

KT group

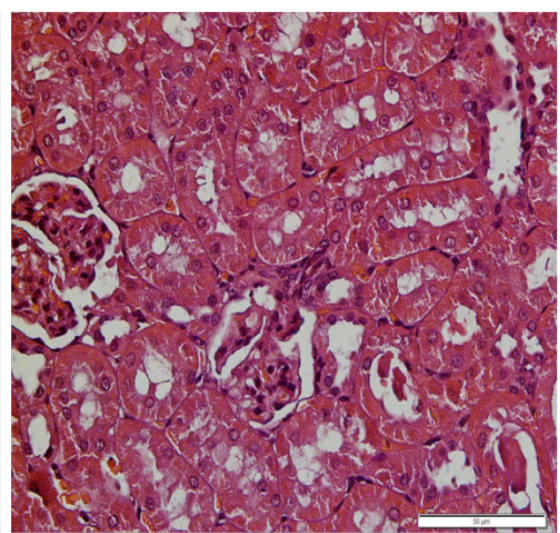

OOP+KT group

Figure 2 - Ozone oxidative preconditioning alleviated the morphological damages after renal transplantation according to the representive micrographs of hematoxylin-eosin staining $(\times 400)$. There were serious morphological lesions in KT group, including renal tubular swelling, necrosis and the distruction of renal tubular normal construction. Furthermore, compared to group KT, morphological damages in group OOP+KT were obviously attenuated. 


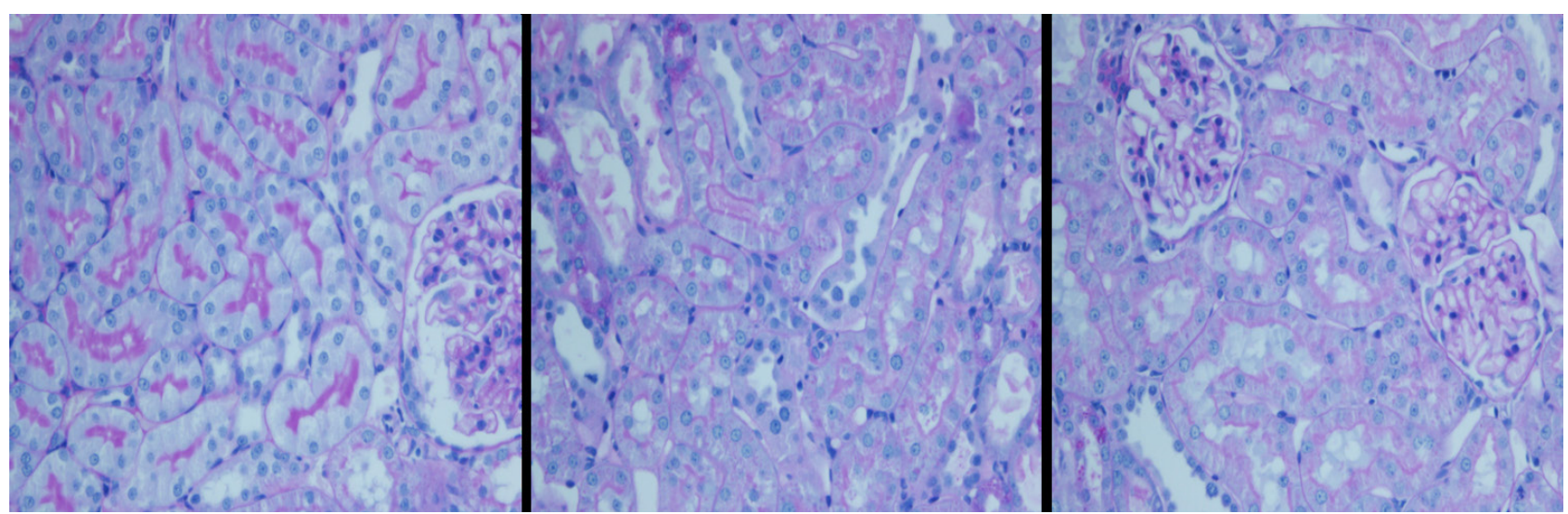

Figure 3 - Ozone oxidative preconditioning attenuated the injury of brush border of proximal renal tubular after kidney transplantation. Representive micrographs of PAS staining showed that severe renal damages including tubular necrosis and distruction of proximal renal tubular brush border were obviously slighter in OOP+KT group than those in KT group $(\times 400)$.

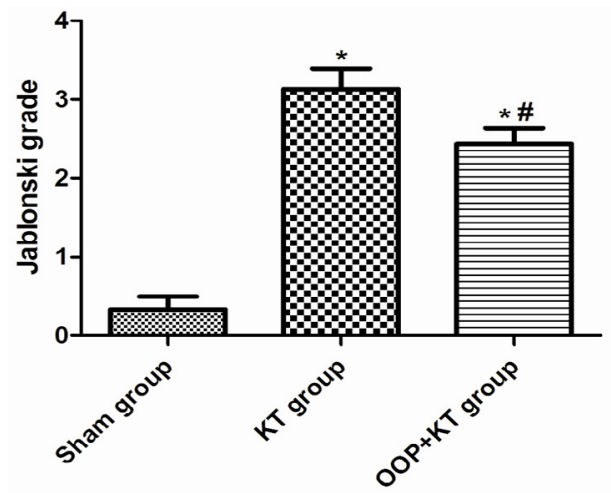

Figure 4 - Ozone oxidative preconditioning reduced the Jablonski grade after kidney transplantation. The Jablonski grades in groups KT and OOP+KT were obviously higher than those in sham group. Furthermore, Jablonski grade in group OOP+KT were significantly lower than that in KT group. ${ }^{*} p<0.05$ vs. sham group, \# $p<0.05 v s$. KT group.

\section{Effect of ozone oxidative preconditioning on the levels of serum inflammatory markers after renal transplantation}

ELISA results showed that serum levels of IL-6, IL-18 and COX-2 in both groups KT and $\mathrm{OOP}+\mathrm{KT}$ were much higher than those in sham group. However, levels of IL-6, IL-18 and COX2 could be significantly down-regulated by ozone oxidative preconditioning according to the results in groups $\mathrm{KT}$ and $\mathrm{OOP}+\mathrm{KT}$ (Figure 5).
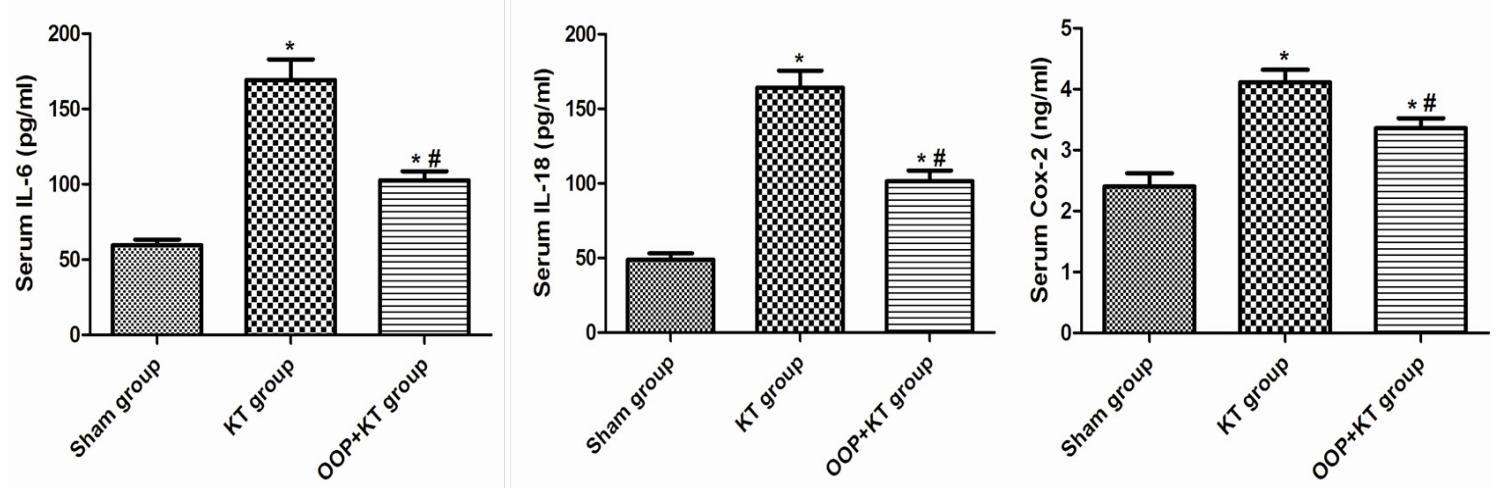

Figure 5 - Ozone oxidative preconditioning down-regulated the level of serum inflammatory factors after kidney transplantation according to the results of ELISA. The levels of serum IL-6, IL-18 and Cox-2 were all significantly higher than those in sham group. Furthermore, compared to group KT, the levels of serum IL-6, IL-18 and Cox-2 in group OOP+KT were all largely decreased. ${ }^{*} p<0.05$ vs. sham group, $\# p<0.05$ vs. KT group. 
Effect of ozone oxidative preconditioning on oxidative stress injury after kidney transplantation

As shown in Table 1, the level of MDA content which was an index of lipid peroxidation was obviously higher in groups KT and OOP+KT than that in sham group. Furthermore, the level of MDA in group OOP+KT was significantly lower than that in group KT. Meanwhile, the levels of SOD and GSH-Px in the kidney tissue were largely lower in KT group than those in sham group. Furthermore, compared to group $\mathrm{KT}$, the reduction of the levels of SOD, GSH-Px after kidney transplantation could be significantly attenuated in OOP+KT group.

Table 1 - Activities of SOD, MDA, GSH-Px in renal tissues.

\begin{tabular}{llll}
\hline Groups & SOD(units/mgprot) & MDA(nmol/mgprot) & GSH-Px (U/mgprot) \\
\hline Sham & $86.24 \pm 7.12$ & $3.52 \pm 1.13$ & $7.61 \pm 0.92$ \\
KT & $38.96 \pm 8.55^{*}$ & $6.48 \pm 1.69^{*}$ & $3.24 \pm 0.85^{*}$ \\
OOP+KT & $56.89 \pm 8.76^{*}, \#$ & $4.92 \pm 1.78^{*}, \#$ & $4.64 \pm 0.59^{*}, \#$ \\
The results are means \pm SD for & & & \\
6 rats in each group. ${ }^{*} \mathrm{p}<0.05$ & & & \\
vs. Sham group , \# $\mathrm{p}<0.05$ vs. KT & & & \\
group. Superoxide Dismutase \\
= SOD; Malonaldehyde = MDA; \\
Glutathione peroxidase = GSH-Px.
\end{tabular}

Effect of ozone oxidative preconditioning on the expression levels of inflammatory markers after renal transplantation

The results of the immunohistochemistry analysis showed that expression levels of NFKBp65 and HMGB1 in OOP+KT group were significantly lower than those in the KT group (Figures 6 and 7). Furthermore, western blot analysis also

Revealed that the expression levels of NF-KBp65 and HMGB1 in OOP+KT group were obviously lower than those in the KT group (Figure 8) $(p<0.05)$.

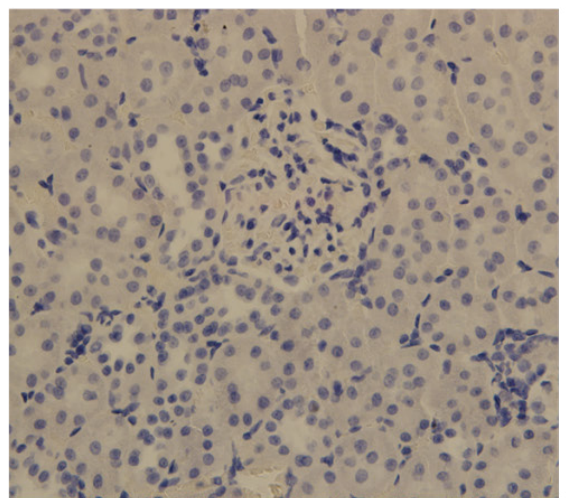

Sham group

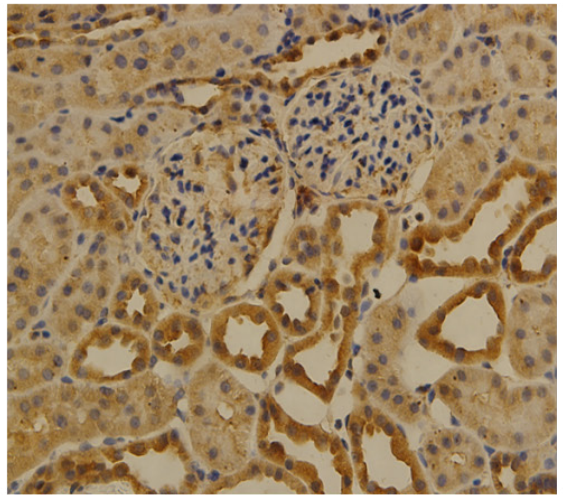

KT group

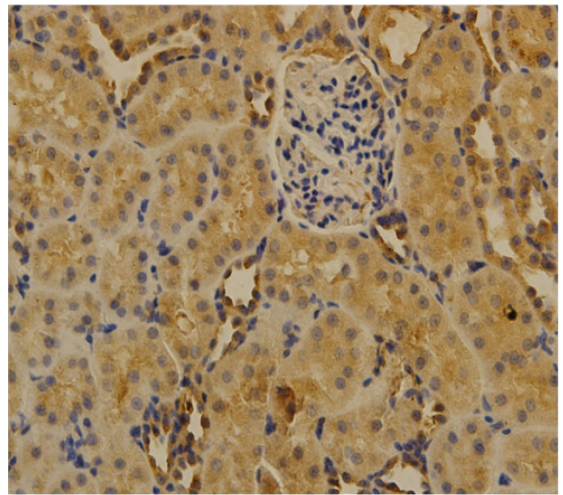

OOP+KT group

Figure 6 - Ozone oxidative preconditioning restrained the expression level of NF- kBp65 in renal tissue after kidney transplantation according to the representive micrographs of immunohistochemistry $(\times 400)$. The expression level of NF-KBp65 in renal tissue were significantly higher in groups KT and OOP+KT than that in sham group. Furthermore, the expression level of NF-KBp65 in group OOP+KT was obviously lower than that in group $\mathrm{KT}$. 


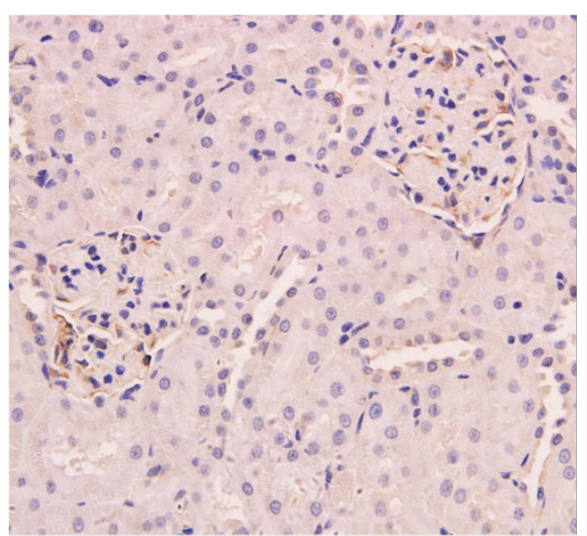

Sham group

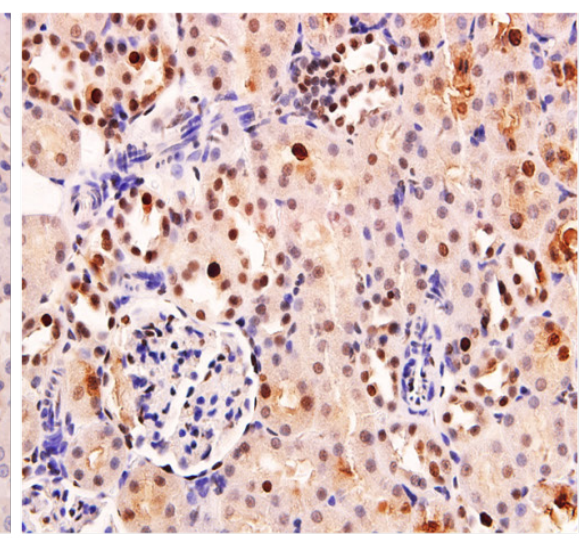

KT group

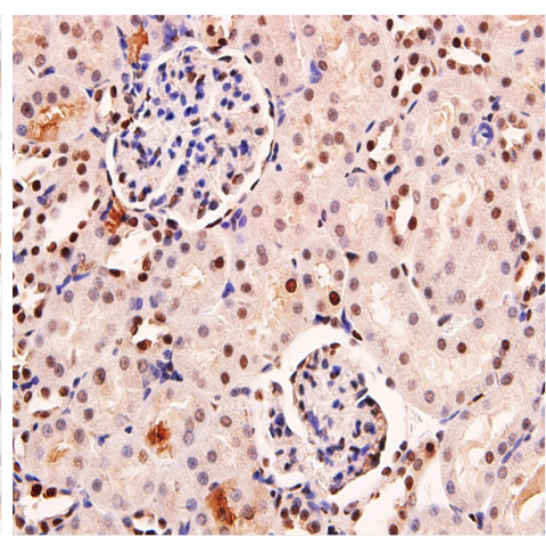

OOP+KT group

Figure 7 - Ozone oxidative preconditioning suppressed the expression of HMGB1 in renal tissue after kidney transplantation according to the representive micrographs of immunohistochemistry $(\times 400)$. The expression level of HMGB1 in renal tissue were obviously higher in groups KT and OOP+KT than that in sham group. Furthermore, the expression level of HMGB1 in group OOP+KT was significantly lower than that in group KT.
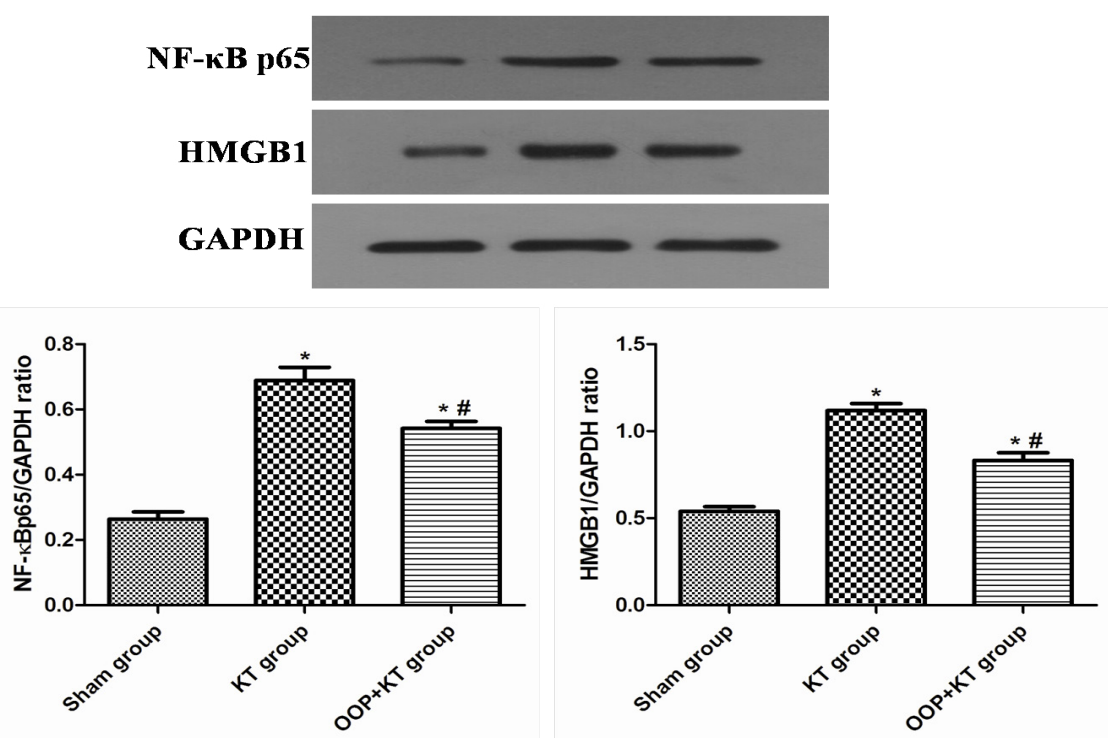

Figure 8 - Ozone oxidative preconditioning inhibited the expression levels of NF-KBp65 and HMGB1 in renal tissue after kidney transplantation according to the results of western blot. The expression levels of NFKBp65 and HMGB1 in renal tissues were obviously higher in groups KT and OOP+KT than those in sham group. Furthermore, both the expression levels of NF-KBp65 and HMGB1 in group OOP+KT were significantly lower than those in group KT. ${ }^{*} p<0.05$ vs. Sham group, $\# p<0.05$ vs. KT group.

\section{- Discussion}

Kidney transplantation provides an effective and important option for the patients who have end-stage renal disease. Furthermore, it has been shown that renal transplantation provide a more considerable improvement in health-related quality of life and a more favorable cost-effectiveness ratio when compared with dialysis ${ }^{5,6}$. Currently, many kinds of immunosuppressive agents which can effectively help to improve the success rate of renal transplantation and graft survival rate. However, there are still some 
unfavourable factors during the perioperative period of kidney transplantation, and one important adverse factor of them is ischemia reperfusion injury, which is hard to avoid.

Ischemia reperfusion injury is involved with a cascade of cellular events which contain cell apoptosis, necrosis, release of reactive oxygen species (ROS), infiltration of inflammatory cells and release of active mediators, and those factors may result in serious injury of tissue. Though the exact mechanisms of ischemia reperfusion injury have not been fully revealed. It has been shown that increasing ROS and pro-inflammatory mediators seems to play an important role during the reperfusion phase ${ }^{7}$. Therefore, reducing the generation or release of ROS and suppressing the release of inflammatory mediators may be benefit for the renal function recovery and long-term survival of renal allograft.

Rodríguez et al. ${ }^{8}$ have shown that ozone as an powerful oxidant gas could promote organ stress by inducing enhancement of endogenous protective parameters such as SOD, CAT, GSH-Px and GSH, in order to alleviate organ injury. In our study, oxidative stress preconditioning could enhance the expression of SOD and GSH-Px in renal tissue after kidney transplantation compared with KT group. These results maybe indicated that ozone oxidative preconditioning could enhance the activity of endogenous anti-oxidant system. Therefore, ozone oxidative preconditioning might help to induce the tolerance to ROS generated by some injury factors or toxic agents, this effect might be analogous to other protective measures such as ischemia preconditioning ${ }^{9}$, chemical preconditioning $^{10}$, thermal preconditioning ${ }^{11}$. It was deserved to show that all these treatments were involved with a repeated and nonlethal stress, which could provide protection against a prolonged and severe stress ${ }^{8}$.
High-mobility group box-1 protein (HMGB1) is a bi-functional protein, which functions as chromatin-associated proteins to regulate transcription in the nucleus. Meanwhile, it can also be released extracellularly to mediate the response to inflammatory stimuli such as infection and injury $^{12}$. It has been identified that HMGB1 could be passively released from necrotic cells and actively secreted by certain cells such as killer cells, monocytes and macrophages ${ }^{13,14}$, which plays a pro-inflammatory role as damage-associated molecular patterns $(\mathrm{DAMPs})^{15}$. It has been reported that Ozone oxidative preconditioning could inhibited the increase in TNF- $\alpha$ production in the model of partial hepatectomy in rats ${ }^{16}$. Furthermore, moderate ozone oxidative stress may also play a role in suppressing NF-KB and inflammatory response ${ }^{17}$. In this study, the expression level of HMGB1 in group OOP+KT was obviously lower than that in KT group. Therefore, the inhibition of HMGB1 production by ozone oxidative preconditioning in the kidney tissue may be one of the factors related to the alleviation of inflammatory response.

Nuclear factor $\mathrm{KB}$ (NF-KB) is a transcription factor that contains five members: P65, P50, P52, RelB, and Rel ${ }^{18,19}$, which plays a key role in the expression of pro-inflammatory cytokine genes ${ }^{20}$. In resting status, NF-KB is an inactive form and sequestered in the cytoplasm by binding the inhibitory protein IKB. Once NF-KB is activated, it will be phosphorylated and ubiquitinated, meanwhile, the inhibitory proteins will be degraded, and the released NF-KB dimers are further activated through modifications and translocated to the nucleus where they bind to specific DNA sequences and thus enhance transcriptional activity of target genes $^{19,21,22}$. Furthermore, León Fernández et $a .^{23}$ have showed that ozone oxidative preconditioning could largely reduced the 
intensity of the p65 expression in the rat model of liver ischemia/reperfusion. Xing et al. ${ }^{24}$ also has showed that ozone oxidative preconditioning had potent anti-inflammatory properties by the modulation of the TLR4NF-KB pathway in renal ischemia/reperfusion injury. In this study, the expression level of NFKBp65 in group OOP+KT is significantly lower than that in group $\mathrm{KT}$, which is coincident with the results of previous researches.

Pro-inflammatory cytokines also play a vital role in the development of kidney injury during the kidney ischemia/reperfusion process. It has been reported that inflammation reaction after kidney ischemia-reperfusion is characterized by upregulation of the production of inflammation cytokines and infiltration of macrophage ${ }^{25}$. IL-6 is an important pleiotropic cytokine, which has been identified that the level of IL-6 in urine correlates with the severity of ischemia-reperfusion injury in human renal allografts ${ }^{26}$. In the present study, the serum level of IL-6 in group OOP+KT was apparently lower than that in group KT. IL-18 is an pleiotropic inflammatory cytokine, whose expression is up-regulated in numerous conditions, such as infection and inflammation ${ }^{27}$. Furthermore, it has been reported that IL-18 was a sensitive and specific biomarker to diagnosis acute kidney injury and predict the mortality of patients ${ }^{28}$. In our study, ozone oxidative preconditioning could largely reduce the level of serum IL-18 in group OOP+KT compared with that in group KT. Another well-know factor is COX-2, which is an inducible enzyme. Research showed that COX2 expressed at sites of infection, inflammation and tumor which generated prostanoids that drive disease pathogenesis ${ }^{29,30}$. It has been showed that COX-2 can be rapidly and robustly expressed in response to diverse range of proinflammation cytokines and mediators ${ }^{31}$. In this study, serum level of COX-2 in OOP+KT group was obviously lower than that in group KT.

\section{Conclusions}

Ozone oxidative preconditioning could attenuated the kidney inflammatory injury and the oxidative stress injury of renal tubular epithelium cells in the rat model of kidney transplantation, which may be related with the inhibitions of NF-kBp65 and HMGB1 upregulation, and reductions of some proinflammatory cytokines. Furthermore, the enhancement of anti-oxidative stress system induced by ozone oxidative preconditioning may be another favour factor which helped to alleviate the kidney ischemia reperfusion injury. However, further research are needed to show the effect of ozone oxidative preconditioning on the model of renal transplantation between different strains rodents or other mammal models, especially the acute immunological rejection model.

\section{References}

1. Bocci VA. Scientific and medical aspects of ozone therapy. State of the art. Arc Med Res. 2006 May;37(4):425-35. PMID: 16624639.

2. Inal M, Dokumacioglu A, Özcelik E, Ucar O. The effects of ozone therapy and coenzyme Q10 combination on oxidative stress markers in healthy subjects. Ir J Med Sci. 2011 Sep;180(3):703-7. PMID: 21258872.

3. Guven A, Gundogdu G, Sadir S, Topal T, Erdogan E, Korkmaz A, Surer I, Ozturk H. The efficacy of ozone therapy in experimental caustic esophageal burn. J Pediatr Surg. 2008 Sep;43(9):1679-84. PMID: 18779006.

4. Jablonski P, Howden BO, Rae DA, Birrell CS, Marshall VC, Tange J. An experimental model for assessment of renal recovery from warm ischemia. Transplantation. 1983 Mar;35(3):198-204. PMID: 6340272.

5. Laupacis A, Keown P, Pus N, Krueger $H$, Ferguson B, Wong C, Muirhead N. A study of the quality of life and cost-utility of renal transplantation. Kidney Int. $1996 \mathrm{Jul} ; 50: 235-$ 42. PMID: 8807593.

6. Schnuelle P, Lorenz D, Trede M, Van Der Woude FJ. Impact of renal cadaveric transplantation on survival in end-stage 
renal failure: evidence for reduced mortality risk compared with hemodialysis during long-term follow-up. J Am Soc Nephrol. 1998 Nov;9:2135-41. PMID: 9808102.

7. Li YW, Zhang Y, Zhang L, Li X, Yu JB, Zhang HT, Tan BB, Jiang LH, Wang YX, Liang Y, Zhang XS, Wang WS, Liu HG: Protective effect of tea polyphenols on renal ischemia/reperfusion injury via suppressing the activation of TLR4/NF-KB p65 signal pathway. Gene. 2014 May 25;542(1):46-51. PMID: 24630969.

8. Rodríguez ZZ, Guanche D, Alvarez RG, Rosales $\mathrm{FH}$, Alonso $\mathrm{Y}$, Schulz S. Preconditioning with ozone/oxygen mixture induces reversion of some indicators of oxidative stress and prevents organic damage in rats with fecal peritonitis. Inflamm Res. 2009 Jul;58(7):3715. PMID: 19274439.

9. Murry CE, Jennings RB, Reimer KA. Preconditioning with ischemia: a delay of lethal cell injury in ischaemic myocardium. Circulation. 1986 Nov;74(5):1124-36. PMID: 3769170.

10.Riepe MW, Ludolph AC. Chemical preconditioning: a cytoprotective strategy. Mol Cell Biochem. 1997 Sep;174(1-2):24954. PMID: 9309696.

11.Neschis DG, Safford SD, Raghunath PN, Langer DJ, David ML, Hanna AK, Tomaszewski JE, Kariko K, Barnathan ES, Golden MA. Thermal preconditioning before rat arterial balloon injury: limitation of injury and surtained reduction of intimal thickening. Arterioscler Thromb Vasc Biol. 1998 Jan;18(1):120-6. PMID: 9445265.

12.Meng $X Y$, Li B, Liu S, Kang $H$, Zhao L, Zhou $R$. EGCG in green tea induces aggregation of HMGB1 protein through large conformational changes with polarized charge redistribution. Sci Rep. 2016 Feb 22;6:22128. PMID: 26899177.

13.Yamasoba D, Tsubota $M$, Domoto $R$, Sekiguchi F, Nishikawa H, Liu K, Nishibori M, Ishikura H, Yamamoto T, Taga A, Kawabata A. Peripheral HMGB1-induced hyperalgesia in mice: Redox state-dependent distinct roles of RAGE and TLR4. J Pharmacol Sci. 2016 Feb;130(2):139-42. PMID: 26883456.

14. He J, Zhang P, Li Q, Zhou D, Liu P. Expression of high mobility group box 1 protein predicts a poorer prognosis for patients with osteosarcoma. Oncol Lett. 2016 Jan;11(1):293-8. PMID: 26870206.
15.Yanai H, Ban T, Taniguchi T. High-mobility group box family of proteins: ligand and sensor for innate immunity. Trends Immunol. 2012 Dec;33(12):633-40. PMID: 23116548.

16.Gultekin FA, Cakmak GK, Turkcu UO, Yurdakan G, Demir FE, Comert M. Effects of ozone oxidative preconditioning on liver regeneration after partial hepatectomy in rats. J Invest Surg. 2013 Oct;26(5):242-52. PMID: 23514050.

17.Sagai M, Bocci V. Mechanisms of action involved in ozone therapy: is healing induced via a mild oxidative stress? Med Gas Res.2011 Dec 20;1:29. PMID: 22185664.

18.O'Sullivan AW, Wang JH, Redmond HP. NF-KB and P38 MAPK inhibitionimprove survival in endotoxin shock and in a cecal ligation and puncture model of sepsis in combination with antibiotic therapy. J Surg Res. 2009 Mar;152(1):46-53. PMID: 19027920.

19. Hayden MS, Ghosh S. Shared principles in NF-KB signaling. Cell. 2008 Feb 8;132(3):34462. PMID: 18267068.

20.Lappas M, Permezel M, Georgiou HM, Rice GE. Nuclear factor kappa B regulation of proinflammatory cytokines in human gestational tissues in vitro. Biol Reprod. 2002 Aug;67(2):668-73. PMID: 12135912.

21.De Stefano D, Maiuri MC, lovine $B$, Ialenti $A$, Bevilacqua MA, Carnuccio R. The role of NF-KB, IRF-1, and STAT-1 $\alpha$ transcription factors in the iNOS gene induction by gliadin and IFN- $\gamma$ in RAW 264.7 macrophages. J Mol Med. 2006 Jan;84(1):65-74. PMID: 16284791.

22.Li W, Wang X, Niu X, Zhang $H$, He Z, Wang Y, Zhi W, Liu F. Protective effects of nobiletin against endotoxic shock in mice through inhibiting TNF- $\alpha, \mathrm{IL}-6$, and HMGB1 and regulating NF-KB pathway. Inflammation. 2016 Apr;39(2):786-97. PMID: 26846885.

23.León Fernández OS, Ajamieh $\mathrm{HH}$, Berlanga $\mathrm{J}$, Menéndez S, Viebahn-Hánsler R, Re L, Carmona AM. Ozone oxidative preconditioning is mediated by $\mathrm{A} 1$ adenosine receptors in a rat model of liver ischemia/ reperfusion. Transpl Int. 2008 Jan;21(1):3948. PMID: 17927680.

24.Xing $B$, Chen $H$, Wang $L$, Weng $X$, Chen $Z$, Li $X$. Ozone oxidative preconditioning protects the rat kidney from reperfusion injury via modulation of the TLR4-NF-KB pathway. Acta Cir Bras. 2015 Jan;30(1):60-6. PMID: 
25627272.

25.Wang J, Long Q, Zhang W, Chen N. Protective effects of exogenous interleukin 18-binding protein in a rat model of acute renal ischemia-reperfusion injury. Shock. 2012 Mar;37(3):333-40. PMID: 22089199.

26.Kwon O, Molitoris BA, Pescovitz M, Kelly KJ. Urinary actin, interleukin-6, and interleukin-8 may predict sustained ARF after ischemic injury in renal allografts. Am J Kidney Dis. 2003 May;41(5):1074-87. PMID: 12722043.

27. Wan X, Yang J, Xing L, Fan L, Hu B, Chen X, Cao $C$. Inhibition of IкB Kinase $\beta$ attenuates hypoxia-induced inflammatory mediators in rat renal tubular cells. Transplant Proc. 2011 Jun;43(5):1503-10. PMID: 21693225.

28.Nisula $S$, Yang $R$, Poukkanen $M$, Vaara ST, Kaukonen KM, Tallgren $M$, Haapio M, Tenhunen J, Korhonen AM, Pettilä V; FINNAKI Study Group. Predictive value of urine interleukin-18 in the evolution and outcome of acute kidney injury in critically ill adult patients. $\mathrm{Br} J$ Anaesth. 2015 Mar;114(3):460-8. PMID: 25472925.

29. Nandakishore R, Yalavarthi PR, Kiran YR, Rajapranathi M. Selective cyclooxygenase inhibitors: current status. Curr Drug Discov Technol. 2014 Jun;11(2):127-32. PMID: 24467591.

30.Kirkby NS, Chan MV, Zaiss AK, Garcia-Vaz E, Jiao J, Berglund LM, Verdu EF, AhmetajShala B, Wallace JL, Herschman HR, Gomez MF,Mitchell JA. Systematic study of constitutive cyclooxygenase-2 expression: Role of NF-KB and NFAT Transcriptional pathways. Proc Natl Acad Sci U S A. 2016 Jan 12;113(2):434-9. PMID: 26712011.

31.Rumzhum NN, Ammit AJ. Cyclooxygenase 2: its regulation, role and impact in airway inflammation. Clin Exp Allergy. 2016 Mar;46(3):397-410. PMID: 26685098.

\section{Correspondence:}

Yong-lian Guo

Department of Urology, The Central Hospital of Wuhan

Tongji Medical College, Huazhong University of Science and Technology

Phone: (86) 027-82211447

guoyl111@126.com

Received: Nov 23, 2017

Review: Jan 25, 2018

Accepted: Feb 22, 2018
Conflict of interest: none

Financial source: none
${ }^{1}$ Research performed at Key Laboratory, Hubei Province for Digestive System Disease, Department of Gastroenterology, Renmin Hospital of Wuhan University, Hubei, China. 